\title{
Reflexiones sobre la ética en la investigación en el proceso formativo de la clínica jurídica "circuitos coloniales del trabajo en el resguardo indígena de San Lorenzo ${ }^{I}$
}

\author{
Reflections about the ethics in research in the training \\ process of the legal clinic "colonial work circuits in the \\ indigenous reserve of San Lorenzo"
}

\section{Elvigia Cardona Zuleta}

Área de Metodología de la Investigación y del Departamento de Prácticas

Facultad de Derecho y Ciencias Políticas.

Universidad de Antioquia, Colombia

E-mail: elvigia.cardona@udea.edu.co

\begin{abstract}
Resumen: Este artículo pretende narrar la experiencia sobre la reflexión de la ética en el trabajo con comunidades étnicas. Se recurre a la investigación cualitativa crítica desde la autorreflexibilidad para interrogarnos e interrogar a esos otros "yo" que nos habitan. Se presentan varias de las preocupaciones con relación a los propósitos de la investigación en la universidad, esto es, responder a las preguntas ¿para qué investigamos? ¿para quién investigamos? y ¿cómo investigamos?, que implican cuestionamientos constantes por la ética en la investigación académica que nos permite evidenciar la potencia de la estrategia de la Clínica Jurídica en la enseñanza-aprendizaje del Derecho y la Ciencia Política posturas responsables y éticas.

Palabras clave: Investigación jurídica y sociojurídica, ética en la investigación, clínica jurídica, comunidades éticas.
\end{abstract}

\footnotetext{
${ }^{1}$ Artículo de reflexión resultado del análisis del Factor Innovación, correspondiente al proceso de Autoevaluación del programa de Derecho, inscrito como proyecto de investigación ante el Centro de Investigaciones de la Facultad, según Acta del Comité Técnico de Investigaciones 04-2015 del 8 de abril de 2015, de la Facultad de Derecho y Ciencias Políticas de la Universidad de Antioquia. El informe de autoevaluación fue entregado en 2016, dando lugar a la reacreditación del pregrado de Derecho mediante Resolución del Ministerio de Educación Nacional No. 7213 del 3 de mayo de 2018. Adicionalmente confluye mi experiencia como profesora e investigadora de la Clínica Jurídica, y de los cursos de Investigación I y II del pregrado de Derecho de la Universidad de Antioquia.
} 


\begin{abstract}
This article aims to narrate the experience on the reflection of ethics in working with ethnic communities. Critical qualitative research is used from selfreflection to question and interrogate those other "I" that inhabit us. Several of the concerns regarding the purposes of university research are presented, that is, answering the questions, why do we investigate? Who do we investigate for? and how do we investigate?, which involve constant questions about ethics in academic research that allows us to demonstrate the power of the strategy of the Legal Clinic in the teachinglearning of Law and Political Science responsible and ethical positions.
\end{abstract}

Keywords: Legal and socio-legal research, research ethics, legal clinic, ethical communities.

\title{
1. Introducción
}

Lo que anima esta investigación ... es el rechazo del conocimiento atomizado, parcelario y reductor, es la reivindicación vital del derecho a la reflexión.

Morin, E. (1980)

Este artículo se presenta de mi participación como profesora e investigadora de la Clínica Jurídica "Circuitos coloniales del trabajo en el Resguardo Indígena de San Lorenzo",2, para el periodo 2014-2016, experiencia que basada en el trabajo colaborativo, nos permitió crear herramientas para impulsar la investigación, sintetizar, orientar y evaluar el trabajo clínico con nuestros estudiantes, además, de permitirnos abordar nuestras preocupaciones sobre perspectivas teóricas, metodológicas y éticas que deben guiar los procesos investigativos en contextos de trabajo comunitario.

La Clínica Jurídica, es una metodología de la enseñanza-aprendizaje del derecho "como un modelo educativo de los estudios jurídicos basado en la conexión con la realidad

\footnotetext{
2 Adscrita al Departamento de Prácticas de la Facultad de Derecho y Ciencias Políticas. El Resguardo Indígena de San Lorenzo, se ubica en el municipio de Caldas-Colombia y allí viven comunidades que se adscriben a la familia Emberá.
} 
social y jurídica" (Blázquez, 2015, p. 5) que propone "replantear la forma de pensar y enseñar el derecho y de hacer del alumno no sólo un receptor sino un interlocutor en el salón de clase" (Rodríguez, 2007, p. 6), en este sentido resumiendo a Courtis (2007) quien propone los siguientes ejes en los cuales debe pensarse este modelo de enseñanza: Eje Pedagógico: i) desarrollo de habilidades para la formulación de la teoría del caso, ii) Trato con el cliente (para nuestro caso trato con las comunidades), iii) destrezas prácticas para abordar los casos. iv) Eje Temático: recuperar temas abandonados por el currículo. v) Eje Profesional: diversas posibilidades del ejercicio de la abogacía, entre ellas la investigación y el trabajo comunitario. vi) Eje Comunitario: Función social de la universidad en la transmisión, producción del conocimiento y transformación de la sociedad.

Así que una de las habilidades y competencia requeridas por los estudiantes es la investigación, lo que nos lleva a una de las frases más conocidas sobre el tema " $A$ investigar se aprende investigando", sin embargo, considero le corresponde también al docente de la clínica jurídica exponer y buscar con los estudiantes las posturas sobre los diversos enfoques, escuelas o corrientes del pensamiento jurídico o sociojurídico, que podemos utilizar para abordar las diferentes investigaciones que emprendemos, las cuales, desembocan en una pluralidad de métodos para responder a nuestras preguntas, que necesariamente tienen que coincidir con nuestra manera de ver el mundo, de aproximarnos a él y de generar conocimiento, además, de interrogarnos sobre nuestras posturas éticas y políticas, frente a las comunidades que intervenimos y a lo esperado por la academia.

Por tanto, "la investigación no es sólo una búsqueda de conocimiento altamente moral y civilizada; es también una serie de actividades humanas que reproducen particulares relaciones sociales de poder." (Smith, 2012, p. 196), relaciones de poder que se han naturalizado en la forma de organizar, conducir y transmitir los resultados de las investigaciones que, de alguna forma, aún hoy, consideran a las personas como objetos de investigación, llegando a vulnerar algunos de sus derechos como el derecho a la información y su autonomía, como puede ocurrir en la investigación con comunidades étnicas.

En este sentido, hoy como investigadoras(es), se nos presenta el reclamo por las finalidades que perseguimos con nuestros trabajos investigativos, esto es, responder a la pregunta ¿para qué investigamos? Son varias las respuestas las que surgen: para cumplir 
con la tarea y ganar una materia, para elaborar un artículo, para cumplir el requisito de trabajo de grado, para cumplir con agendas nacionales y universitarias de investigación, para nuestro propio goce... o estamos comprometidos con transformar la sociedad y avanzar en la justicia social de nuestros propios entornos, en este sentido cobra importancia reflexionar sobre la investigación jurídica, la ética en la investigación y el proceso formativo de la Clínica Jurídica circuitos coloniales en el Resguardo Indígena de San Lorenzo, aunque de manera breve y sin pretensiones de ser exhaustiva, presento a continuación estos pensamientos surgidos en los ires y venires, entretejiendo nuestros saberes, nuestras preocupaciones, logros y desencuentros en la enseñanza-aprendizaje del Derecho.

En términos metodológicos recurro a la investigación cualitativa, a partir de la autorreflexibilidad, entendida como "experimentar el self en forma consciente" (Denzin y Lincoln, 2012, p. 67) que rompe con las jerarquías en el desarrollo del trabajo colaborativo que se lleva a cabo en la Clínica Jurídica, como estrategia de enseñanzaaprendizaje, y me permite asumir una postura ética y política como investigadora y la confluencia de mis experiencias cotidianas de los otros "yo" que me habitan, la mujer, la profesora, la egresada y la compañera de trabajo, de esta forma "cada uno de esos selves entra en juego en el entorno de la investigación y, por consiguiente, tiene voz distintiva." (p. 67)

\section{Reflexión sobre la ética de la investigación en las comunidades étnicas}

"La ética no está al final, ni es un curso.

La ética por sí posibilita la educación, una educación que no concluye.

"Yo soy tú, cuando yo soy yo","

\section{Joan Carles Mélich}

Abordar la reflexión por la ética en la investigación cualitativa parte de las preguntas ¿Podemos causar daño con nuestras investigaciones? ¿Qué tipos de daños podemos causar con nuestras investigaciones? Sin embargo, entre la investigación cualitativa en el área del derecho y la procedencia de los principios éticos en la investigación parecía existir un vacío para la disciplina jurídica, puesto que tradicionalmente la investigación jurídica y sociojurídica se veía influida por "una enseñanza jurídica discursiva, 
memorística y repetitiva que omite todo juicio crítico y participativo de estudiantes pasivos y esencialmente receptivos" (Witker, 2008, p. 944)

En este sentido, en Colombia, autores en el campo de la metodología de la investigación jurídica $^{3}$, no incluyen en sus tratados sobre investigación cualitativa jurídica o sociojurídica apartados sobre los aspectos éticos, pero el avance científico, los cambios en la metodología de enseñanza aprendizaje propuestos por la Clínica Jurídica, el movimiento en la concepción del objeto de estudio del derecho entendido como cultura, la creciente necesidad en avanzar en investigaciones empíricas, holísticas e integrativas (Witker, 2008) que permitan explicar los fenómenos jurídicos, además de las normativas internacionales y nacionales sobre la ética en la investigación, dan lugar a incluir en nuestras investigaciones los aspectos éticos, no como un mero requisito o acápite del formato para la formulación del proyecto de investigación, sino en respuesta a los cuestionamientos sobre qué es lo éticamente aceptable cuando se adelanta investigación en el área académica que involucra comunidades.

En Colombia, en el año 2010 se crea el Consejo Nacional de Bioética, con la finalidad de "establecer un diálogo interdisciplinario para formular, articular y resolver los dilemas que plantea la investigación (...), así como la construcción e implementación de políticas en los asuntos referentes a la Bioética.” (art. 2) y años después expide la Política de Ética, Bioética e Integridad Científica (Colciencias, 2017), incorporada al ordenamiento jurídico mediante Resolución 314 de 2018, en la que se evidencia la ausencia de los Comités de Ética en las instituciones académicas, la normatividad fragmentada y el bajo seguimiento a los efectos de las investigaciones que se adelantan.

Por su parte, la Universidad de Antioquia mediante Resolución Rectoral 40455 crea el Comité Central de Ética en Investigación de la Universidad de Antioquia -CCEI y mediante documento publicado en 2015 el Manual para el funcionamiento de los Comités de Ética en Investigación de la Universidad de Antioquia (MCE), con lo cual se formalizan y unifican los marcos normativos de los comités ${ }^{4}$ que venían funcionando para las áreas médicas y de la salud. En los últimos dos años en las convocatorias que hace la Universidad mediante el Sistema Universitario de Investigación y el Comité

\footnotetext{
${ }^{3}$ Tales como, Jaime Giraldo Ángel (2012) y sus libros Metodología y Técnica de la Investigación Jurídica y Metodología y Técnica de la Investigación Sociojurídica o Joaquín Andrés Gallego Marín (2012) y su libro Cuadernos de metodología de la investigación para la escuela de derecho: Líneas básicas para direccionar el qué hacer de la investigación, por mencionar algunos.

${ }^{4}$ Desde el 2003 se crea el Comité de Bioética, y para 2014 funcionan 6 Comités de Ética establecidos para las diferentes especialidades médicas, de la salud, de farmacia, experimentación con animales y ciencias sociales.
} 
para el Desarrollo de la Investigación dirigidas a profesores y las realizadas por la Facultad de Derecho y Ciencias Políticas, dirigidas a estudiantes, incluyen un apartado en las propuestas denominado "componente ético" extensiva a todas las áreas del conocimiento.

Con este marco normativo se busca "el desarrollo de un plan de mejoramiento que contribuya con procesos de formación para la comunidad académica y con la consolidación de una cultura de buenas prácticas investigativas.” (R40455, 2014, p. 1) con la cual se establecen "procesos autoevaluativos al interior de las comunidades académicas y heteroevaluativos, mediante pares y comités relacionados con los aspectos científico-técnicos y éticos de la investigación.” (R40455, 2014, p. 1).

Los principios básicos que deben contemplarse por parte de los Comités de Ética de Investigación (CEI) deben atender según los lineamientos establecidos en el Manual de los Comités de Ética de Investigación en la Universidad de Antioquia a:

Los CEI fundarán sus actuaciones en la recta ponderación, interpretación y aplicación de aquellos principios que permitan salvaguardar la protección de la biosfera y de los derechos y bienestar de los seres vivos humanos y no humanos involucrados directa o indirectamente en las investigaciones que se realizan en la institución; principalmente los principios de responsabilidad, transparencia, autonomía, independencia, profesionalidad, no maleficencia, justicia, respeto a las personas y a la biodiversidad, beneficencia, multiculturalidad y vulnerabilidad. (MCE, 2015, p. 6).

Sin embargo, más allá de los criterios objetivos establecidos por los comités de ética institucionales y los principios que deben regir las actuaciones de los investigados, éstos, deben asumir posturas éticas para el desarrollo de sus investigaciones, las cuales se traducen en la práctica de manera diferente, a partir de la perspectiva asumida para sus investigaciones, así, al partir de paradigmas de investigación positivistas y postpositivistas, los comités se convierten en meros requisitos que deben ser cumplidos para obtener la financiación por parte de la Universidad y de Colciencias, pero al acercarse a paradigmas críticos, constructivistas y participativos según clasificación de Denzin (2012), donde el investigador asume una postura ética y política.

En este sentido, partimos de comprender la ética como algo inherente al ser humano y su condición social "una persona puede ser más moral o menos según determinados códigos... Es lo que algunos filósofos han querido decir al afirmar que no hay seres humanos amorales, situados más allá del bien y del mal" (Cortina, 2016, p. 7) y del enfoque de acción sin daño, entendido como: 
La Acción sin Daño (Do no Harm), parte de la premisa de que las organizaciones de cooperación, agencias de desarrollo y ayuda humanitaria, así como pueden contribuir a transformar positivamente situaciones de conflicto, mejorar las condiciones de vida de personas vulnerables y ayudar a cerrar brechas sociales, entre otros, también pueden generar daño, aumentando las tensiones, fragmentando aún más el tejido social o exacerbando los conflictos. (Confederación Suiza, sf, p. 1).

La reflexión que el investigador debe incluir desde el diseño de su investigación, permite de un lado ser consciente, señalar, y enfrentar los dilemas éticos que pueden surgir en el trabajo comunitario, y de otro lado entender cuáles son las limitaciones, restricciones y exclusiones de las actuaciones del investigador, su equipo de trabajo y las relaciones individuales o colectivas que se entablan al interior de la comunidad, sin olvidar que en el marco de la clínica jurídica no se puede descuidar la relación con los estudiantes, ya que los dilemas éticos se presentan tanto en las relaciones investigadores-comunidad, como en las relaciones profesores-estudiantes.

El primer paso entonces, (desde el diseño de la investigación) es recordar los 3 principios propuestos por la Comisión Nacional para la Protección de los Sujetos Humanos de Investigación Biomédica y del Comportamiento, en el denominado Informe Belmont (1979) que hoy cobra plena vigencia:

(1) respeto a las personas (...) todos los individuos deben ser tratados como agentes autónomos, y la segunda, que todas las personas cuya autonomía está disminuida, tienen derecho a ser protegidas (p. 11)

(2) beneficencia, entendida como “(1) No causar ningún daño, y (2) maximizar los beneficios posibles y disminuir los posibles daños.” (p. 3)

(3) justicia - en el sentido de "equidad en la distribución" (...) de las cargas y beneficios. (p. 4).

El segundo paso es comprender cuáles son aquellas acciones que deben desplegarse para mitigar o minimizar el daño derivado de las malas prácticas en la investigación que se dan al desconocer estos principios, para ello el programa CITI Ética en la Investigación de la Universidad de Miami (2012), propone que se elabore una medición desde el diseño de la investigación en la cual se tomen en cuenta: el Análisis de Vulnerabilidad y el Análisis del Riesgo.

En primer lugar, la vulnerabilidad está asociada a las condiciones del ser humano su dignidad, su individualidad e intimidad y la posibilidad real de hacerle daño, además de ese entrecruzamiento con situaciones del orden económico, social, ambiental, cultural, político y educativo, que hacen que una persona o grupo de personas se denominen 
como "poblaciones vulnerables, para referirse a aquellos grupos de personas que, a consecuencia de las condiciones del medio en que viven, están en una situación de mayor susceptibilidad al daño" (Feito, 2007, p. 2) de allí el llamado internacional a la protección y respeto a la integridad humana. (Unesco, 2005)

A partir de esta definición, estamos de acuerdo en el equipo de trabajo de la clínica jurídica, de que nos enfrentamos en un contexto de alta vulnerabilidad, puesto que como lo reconoce la Declaración Universal sobre Bioética y Derechos Humanos: "la conducta científica y tecnológica poco ética ha tenido repercusiones especiales en las comunidades indígenas y locales" (Unesco, 2005, p. 1), es decir, que las comunidades étnicas, internacionalmente son reconocidos como población en situación de especial vulnerabilidad, en el sentido de que frente a la ocurrencia de un perjuicio pueden verse afectados en mayor grado que otros grupos de personas.

Sumado a esto, en conversaciones informales con algunas personas de la comunidad, nos señalaron su desacuerdo con los procesos investigativos adelantados, porque: "no tienen en cuenta nuestros ciclos vitales", "no comparten los resultados obtenidos" y "en varias ocasiones han visto publicados resultados sin que ellos se enteraran de ser el objeto de investigación" pero además, se puede señalar la existencia de múltiples y complejas vulnerabilidades, puesto que se pretende adelantar una investigación que involucra a mujeres trabajadoras, mujeres perseguidas por la ley penal y mujeres menores de edad, de allí que nuestro accionar puede llegar a causar: pérdidas económicas, del empleo, tensiones sexo-genéricas y tensiones frente a las prácticas culturales reconocidas y arraigadas en la comunidad.

Las acciones que tomamos para reducir los posibles daños derivados de esta situación y acorde con las recomendaciones del programa CITI Investigación con grupos vulnerables en la cual se recomienda: Consulta a la Comunidad, Evaluación colaboradora para el comité de ética institucional, Plan de Consulta para el seguimiento, Plan para informar avances y resultados (CITI, 2012, lección 20), en primer lugar frente a la Consulta a la Comunidad, nos articulamos al Consultorio Jurídico Rural, quienes contaban con invitación realizada por el Gobierno Indígena, en el sentido de apoyarlos en la atención jurídica individual y al programa sociojurídico QIRISIA QAWAI, quienes desde el semestre 2014-2, avanzaban en procesos de Formación Jurídica y Política Básica, proyectos de investigación e intervención, ya que viajaban cada quince días al Resguardo a partir del convenio realizado entre la Gobernación Indígena de San 
Lorenzo y la Facultad de Derecho y Ciencias Políticas, así, en la programación del semestre 2016-1, se conformó un espacio de trabajo con la comunidad en la cual se cumple con el principio de reciprocidad en términos de abordar temas de interés comunitarios denominado "Identidad y ser indígena en San Lorenzo" y a partir de allí avanzar en los diferentes proyectos de investigación, logrando una articulación de esfuerzos, que en primer lugar beneficie a la comunidad y optimice los recursos.

En cuanto a la actividad descrita como Evaluación colaboradora para el Comité de Ética Institucional, vale la pena señalar que la Facultad de Derecho y Ciencias Políticas, aún no cuenta con un Comité de Ética en Investigación, y que los aspectos éticos se exigen para aquellas investigaciones con financiamiento institucional, por lo que ejercicios de aula, como los propuestos en la clínica jurídica, quedarían por fuera de la reglamentación de los Comités y los Códigos vigentes.

Sin embargo, se opta en la asesoría que se brinda a los estudiantes para adelantar el proyecto, seguir rigurosamente con los principios éticos y así se decide incluir en el cronograma los planes de seguimiento y evaluación de cada una de las actividades por parte de la comunidad, además de dos visitas al finalizar la recolección y generación de datos a fin de validar la información con la comunidad, en un acto de respeto y de reconocimiento de que el conocimiento puede ser construido socialmente y no le pertenece exclusivamente a la academia.

El tercer paso, se refiere al análisis del riesgo, es necesario tener en cuenta dos variables que requieren ser evaluadas: la probabilidad de ocurrencia y la magnitud del daño. De esta manera:

\footnotetext{
Al evaluar el riesgo asociado con la participación en un estudio de investigación, es necesario considerar dos elementos diferentes de riesgo. Uno es la probabilidad de que un daño específico pueda ocurrir. No todos los daños posibles son igual de probables, y este hecho debería tenerse en consideración al evaluar el riesgo. El segundo elemento de riesgo es la magnitud de tal daño. Algunas veces existe una gran disparidad entre la probabilidad y la magnitud del daño en un estudio. (CITI, año, lección 10).
}

Para esta evaluación se opta por elaborar una matriz, que permita identificar el riesgo asociado, la probabilidad de que ocurra en una graduación baja, media, alta y la magnitud del daño con una graduación que también se gradúa en la misma escala, así: 


\section{Cuadro 1. Matriz Análisis de Riesgos}

\begin{tabular}{|c|c|c|c|}
\hline Descripción del Riesgo & $\begin{array}{c}\text { Probabilidad de } \\
\text { ocurrencia }\end{array}$ & $\begin{array}{l}\text { Magnitud del } \\
\text { daño }\end{array}$ & $\begin{array}{l}\text { Acciones para mitigar, } \\
\text { corregir y evitar }\end{array}$ \\
\hline $\begin{array}{l}\text { Grupo originario, mujeres y } \\
\text { menores de edad, son grupos } \\
\text { internacionalmente } \\
\text { protegidos }\end{array}$ & $\begin{array}{l}\text { Baja: Se parte del } \\
\text { principio de respeto } \\
\text { por las personas y } \\
\text { de la Comunidad }\end{array}$ & $\begin{array}{l}\text { Baja: El } \\
\text { investigador debe } \\
\text { estar atento para } \\
\text { evitar trasgredir } \\
\text { este derecho }\end{array}$ & $\begin{array}{l}\text { Se cuenta con la autorización } \\
\text { de la Gobernación Indígena, } \\
\text { a quien el Coordinador de la } \\
\text { Práctica, le entrega informes } \\
\text { semestrales en el marco del } \\
\text { convenio que tiene con la } \\
\text { Facultad de Derecho. } \\
\text { Adicionalmente previo a la } \\
\text { publicación se presentarán } \\
\text { borradores, para la validación } \\
\text { y autorización de publicación } \\
\text { del Gobierno Indígena. }\end{array}$ \\
\hline $\begin{array}{c}\text { Tensiones frente a la Cultura } \\
\text { y prácticas arraigadas }\end{array}$ & $\begin{array}{l}\text { Alta: Se requiere } \\
\text { partir del respeto } \\
\text { por los derechos de } \\
\text { los pueblos } \\
\text { originarios }\end{array}$ & $\begin{array}{c}\text { Alta: La } \\
\text { investigación } \\
\text { puede generar } \\
\text { mayor } \\
\text { estigmatización o } \\
\text { tergiversar las } \\
\text { prácticas }\end{array}$ & $\begin{array}{l}\text { El equipo de trabajo debe } \\
\text { revisar posturas teóricas } \\
\text { Interculturales, Decoloniales, } \\
\text { de Género y de Ecología de } \\
\text { Saberes que permita entender } \\
\text { y darle voz a la comunidad, } \\
\text { buscando la construcción del } \\
\text { conocimiento en conjunto y } \\
\text { el reconocimiento de su } \\
\text { conocimiento ancestral. }\end{array}$ \\
\hline Invasión de Privacidad & $\begin{array}{c}\text { Baja: Se parte del } \\
\text { principio de respeto } \\
\text { por las personas y } \\
\text { de la no } \\
\text { obligatoriedad de } \\
\text { participar. }\end{array}$ & $\begin{array}{c}\text { Baja: El } \\
\text { investigador debe } \\
\text { estar atento para } \\
\text { evitar trasgredir } \\
\text { este derecho }\end{array}$ & $\begin{array}{l}\text { Utilizar procedimientos para } \\
\text { el consentimiento informado } \\
\text { individual. } \\
\text { Escritura de borradores y } \\
\text { validación con las personas } \\
\text { participantes de entrevistas y } \\
\text { talleres. }\end{array}$ \\
\hline Ruptura de Confidencialidad & $\begin{array}{l}\text { Baja: Se establecen } \\
\text { procedimientos } \\
\text { para categorizar y } \\
\text { codificar }\end{array}$ & $\begin{array}{l}\text { Alta: Revelar } \\
\text { información } \\
\text { puede acarrear } \\
\text { estigmatización y } \\
\text { daños a no } \\
\text { participantes }\end{array}$ & $\begin{array}{l}\text { Codificación de la } \\
\text { información y asignación de } \\
\text { clave alfanumérica al } \\
\text { entrevistado que quiere } \\
\text { permanecer en anonimato }\end{array}$ \\
\hline $\begin{array}{c}\text { Expectativas de solución a la } \\
\text { problemática por parte de la } \\
\text { Comunidad }\end{array}$ & $\begin{array}{l}\text { Alta: Por la } \\
\text { Confianza en la } \\
\text { Facultad de } \\
\text { Derecho y Ciencias } \\
\text { Políticas }\end{array}$ & $\begin{array}{l}\text { Alta: Se puede } \\
\text { afectar la imagen } \\
\text { de la UdeA }\end{array}$ & $\begin{array}{l}\text { En el consentimiento } \\
\text { informado y en cada visita } \\
\text { hacer explícito que se está } \\
\text { haciendo un trabajo } \\
\text { académico y que se requieren } \\
\text { alianzas para proponer e } \\
\text { impulsar cambios. }\end{array}$ \\
\hline $\begin{array}{l}\text { Resistencia y Reactividad de } \\
\text { personas en la Comunidad }\end{array}$ & $\begin{array}{l}\text { Media: Por la } \\
\text { confianza en la } \\
\text { FDCP }\end{array}$ & $\begin{array}{l}\text { Alta: Las } \\
\text { personas han } \\
\text { manifestado estar } \\
\text { cansadas de los } \\
\text { investigadores } \\
\text { que no aportan a } \\
\text { la solución de }\end{array}$ & $\begin{array}{l}\text { Ganar confianzas a partir del } \\
\text { cumplimiento de } \\
\text { compromisos que puedan } \\
\text { adquirirse en la esfera de lo } \\
\text { que sea posible por parte de } \\
\text { los investigadores. Generar } \\
\text { sinergias y articulaciones que }\end{array}$ \\
\hline
\end{tabular}




\begin{tabular}{|c|c|c|c|}
\hline Descripción del Riesgo & $\begin{array}{l}\text { Probabilidad de } \\
\text { ocurrencia }\end{array}$ & $\begin{array}{l}\text { Magnitud del } \\
\text { daño }\end{array}$ & $\begin{array}{l}\text { Acciones para mitigar, } \\
\text { corregir y evitar }\end{array}$ \\
\hline & & problemas & $\begin{array}{l}\text { permitan mayor } \\
\text { financiamiento. }\end{array}$ \\
\hline $\begin{array}{l}\text { Identificar vulneraciones a } \\
\text { menores de edad, en el caso } \\
\text { de las niñas empleadas del } \\
\text { servicio doméstico. }\end{array}$ & $\begin{array}{c}\text { Alta: Si se aplica en } \\
\text { rigor la Ley } \\
\text { 1098/2006 que } \\
\text { exige } \\
\text { corresponsabilidad. }\end{array}$ & $\begin{array}{c}\text { Alta: Se pueden } \\
\text { acarrear pérdidas } \\
\text { económicas para } \\
\text { las familias que se } \\
\text { ven remuneradas } \\
\text { por el trabajo de } \\
\text { niñas menores de } \\
14 \text { años y } \\
\text { procesos de } \\
\text { Restablecimiento } \\
\text { de Derechos }\end{array}$ & $\begin{array}{l}\text { Establecer sinergias con el } \\
\text { Consultorio Jurídico Rural de } \\
\text { San Lorenzo para la atención } \\
\text { individual. Solicitar } \\
\text { autorizaciones y poner en } \\
\text { conocimiento de las } \\
\text { Autoridades Indígenas. } \\
\text { Evitar publicar información }\end{array}$ \\
\hline $\begin{array}{l}\text { Incremento de tensiones } \\
\text { sexo-genéricas }\end{array}$ & $\begin{array}{l}\text { Alta: La comunidad } \\
\text { es tradicionalmente } \\
\text { patriarcal y el } \\
\text { trabajo remunerado } \\
\text { de las mujeres } \\
\text { genera } \\
\text { independencia. }\end{array}$ & $\begin{array}{c}\text { Media: Es } \\
\text { evidente, algunas } \\
\text { mujeres participan } \\
\text { del gobierno } \\
\text { indígena. }\end{array}$ & $\begin{array}{l}\text { En los Talleres que se } \\
\text { adelanten, tener un enfoque } \\
\text { de género e invitar a la } \\
\text { participación tanto a } \\
\text { hombres, como a mujeres } \\
\text { jóvenes y adultos, para } \\
\text { generar posturas genero- } \\
\text { sensitivas. }\end{array}$ \\
\hline $\begin{array}{l}\text { Pérdidas económicas y del } \\
\text { empleo }\end{array}$ & $\begin{array}{c}\text { Baja: Se está en } \\
\text { una fase } \\
\text { exploratoria }\end{array}$ & $\begin{array}{c}\text { Alta: es posible } \\
\text { identificar } \\
\text { situaciones } \\
\text { contrarias a la } \\
\text { legislación laboral } \\
\text { aplicable. }\end{array}$ & $\begin{array}{c}\text { Articularse con el programa } \\
\text { Qirisia quienes adelantan } \\
\text { proyectos productivos y con } \\
\text { el proyecto de la Escuela de } \\
\text { Gobierno, incluir formación } \\
\text { jurídica y política básica en } \\
\text { términos de Derechos } \\
\text { Laborales }\end{array}$ \\
\hline $\begin{array}{l}\text { Ocultamiento o Engaño en } \\
\text { los procesos investigativos }\end{array}$ & $\begin{array}{l}\text { Baja: Por la actitud } \\
\text { del equipo de } \\
\text { Clínica Jurídica y } \\
\text { las convicciones } \\
\text { compartidas sobre } \\
\text { la ética y la política } \\
\text { en la Investigación }\end{array}$ & $\begin{array}{c}\text { Bajo: El equipo } \\
\text { de Clínica } \\
\text { Jurídica es } \\
\text { reconocido, } \\
\text { además, se cuenta } \\
\text { con el apoyo del } \\
\text { profesor Jader } \\
\text { Suaza Estrada } \\
\text { quien ha ganado } \\
\text { confianzas en la } \\
\text { comunidad }\end{array}$ & $\begin{array}{c}\text { El equipo de Clínica Jurídica } \\
\text { trabaja de manera } \\
\text { colaborativa con el programa } \\
\text { sociojurídico Qirisia, con la } \\
\text { finalidad de que la } \\
\text { comunidad no perciba que } \\
\text { existen diferentes grupos } \\
\text { investigando, sino que todos } \\
\text { pertenecemos a la Facultad } \\
\text { de Derecho y Ciencias } \\
\text { Políticas. En el espacio } \\
\text { formativo "Identidad y Ser } \\
\text { Indígena en San Lorenzo" se } \\
\text { adelantan los talleres que } \\
\text { permiten recoger y generar } \\
\text { información con y desde la } \\
\text { comunidad, para todos los } \\
\text { estudiantes que adelantan } \\
\text { diversos proyectos de } \\
\text { investigación. }\end{array}$ \\
\hline Invención/Falsificación & $\begin{array}{l}\text { Baja: Por la actitud } \\
\text { del equipo de }\end{array}$ & $\begin{array}{l}\text { Baja: Control y } \\
\text { Evaluación }\end{array}$ & $\begin{array}{l}\text { Se exige para cada taller que } \\
\text { se elabore guía que incluya la }\end{array}$ \\
\hline
\end{tabular}




\begin{tabular}{|c|c|c|c|}
\hline Descripción del Riesgo & $\begin{array}{l}\text { Probabilidad de } \\
\text { ocurrencia }\end{array}$ & $\begin{array}{l}\text { Magnitud del } \\
\text { daño }\end{array}$ & $\begin{array}{c}\text { Acciones para mitigar, } \\
\text { corregir y evitar }\end{array}$ \\
\hline & $\begin{array}{l}\text { Clínica Jurídica y } \\
\text { las convicciones } \\
\text { compartidas sobre } \\
\text { la ética y la política } \\
\text { en la Investigación }\end{array}$ & $\begin{array}{l}\text { permanente por } \\
\text { parte de los } \\
\text { asesores y de los } \\
\text { estudiantes que } \\
\text { adelantan } \\
\text { proyectos }\end{array}$ & $\begin{array}{l}\text { descripción de la actividad, } \\
\text { los recursos necesarios y la } \\
\text { evaluación. Listado de } \\
\text { asistencia y consentimiento } \\
\text { informado. Relatoría } \\
\text { posterior a la actividad con } \\
\text { evidencias fotográficas. } \\
\text { Categorización de la } \\
\text { información. }\end{array}$ \\
\hline Plagio & $\begin{array}{l}\text { Baja: Por la actitud } \\
\text { del equipo de } \\
\text { Clínica Jurídica y } \\
\text { las convicciones } \\
\text { compartidas sobre } \\
\text { la ética y la política } \\
\text { en la Investigación }\end{array}$ & $\begin{array}{l}\text { Baja: Control y } \\
\text { Evaluación } \\
\text { permanente por } \\
\text { parte de los } \\
\text { asesores y de los } \\
\text { estudiantes que } \\
\text { adelantan } \\
\text { proyectos }\end{array}$ & $\begin{array}{l}\text { Compromiso con la } \\
\text { rigurosidad en la citación y } \\
\text { otorgamiento de los créditos } \\
\text { respectivos a estudiantes, } \\
\text { autores consultados y la voz } \\
\text { de las personas participantes. } \\
\text { Evitar la tergiversación de } \\
\text { información, para ello se } \\
\text { hace validación con la } \\
\text { comunidad. }\end{array}$ \\
\hline
\end{tabular}

Fuente: elaboración propia.

Recapitulando, es necesario entonces, prestar atención a los posibles riesgos que se derivan de las investigaciones que adelantamos, la invitación es a tener presente acciones y estrategias que permitan de un lado entender los riesgos a los que nos enfrentamos y de otro, hacer todo lo posible por mitigarlos y recordar la importancia del Consentimiento informado, la información clara precisa y verás que se debe ofrecer a las comunidades sobre los beneficios y posibles riesgos y el respeto a las personas.

Derivado de esto optamos en las asesorías por hacer una evaluación constante de los riesgos que es necesario considerar y verificación de las acciones que se deben adelantar para evitarlos, en las que se cruzan las variables de vulnerabilidad, con nivel o alcance del daño y posibilidad de ocurrencia, así que cuando el cruce es (daño alto, posibilidad de ocurrencia máxima) se consideran investigaciones que por su magnitud en el daño y su máxima probabilidad de ocurrencia, es mejor no adelantar, atendiendo al sentido ético y responsabilidad social universitaria.

\section{Consideraciones Finales}

Para concluir, la clínica jurídica, se ha configurado como un espacio para la educación intercultural, centrada en el diálogo, con los limitantes de tiempo que imponen los semestres académicos 5 , la ausencia de recursos económicos ${ }^{6}$ y las dificultades que se

\footnotetext{
${ }^{5}$ Sobre el detalle de actividades y resultados de la Clínica Jurídica, pueden consultarse los siguientes informes de gestión: Informe 2015-Unico, Informe 2016-1, Informe 2016-2
} 
generan por el contexto político de la Universidad de Antioquia, ${ }^{7}$ pese a estos factores, podemos resaltar los siguientes aspectos, del trabajo realizado, el cual apenas comienza, ya que si la intensión es lograr un litigio de alto impacto, necesitamos primero, investigar, reflexionar, explorar esta problemática desde otras lógicas, otras teorías, otros lugares del conocimiento, lo que nos permite articular los ejes de la clínica jurídica, así:

Desde el eje ético: Se promueve en el equipo clínico los principios éticos transversales en las relaciones profesores-estudiantes y estudiantes-comunidad, así como el respeto a las comunidades y personas que intervienen en nuestras investigaciones, independiente de que sea un requisito objetivo de presentación de propuestas para obtener financiamiento, o de que exista una normatividad interna o nacional que lo exija. Los asuntos éticos están ligados a las cualidades personales "labrarse un buen carácter, un buen ethos, es lo más inteligente que puede hacer una persona para aumentar sus posibilidades de llevar a cabo una vida buena, feliz." (Cortina, 2016, p. 45) y al reconocimiento del otro, como ser importante y aportante a la construcción social del conocimiento.

Desde eje pedagógico: Se lograron establecer relaciones horizontales entre estudiantes y profesores, de acogimiento, responsabilidad y hospitalidad (Bárcena y Mélich, 2000) se evidencia como ya no es posible repetir un discurso aprendido, porque lo que nos ha enseñado el trabajo clínico, es que una misma problemática, cuando reconocemos que nuestros estudiantes traen un bagaje académico, personal y contextual, desde sus lecturas individuales, su manera de ver el mundo y afrontarlo, pueden aportar nuevas perspectivas e interpretaciones de los viejos problemas, por lo que nuestra tarea será apoyarlos y acompañarlos en sus iniciativas y aprender con ellos, en esas rutas que se van tejiendo en el aprendizaje.

Desde el eje de destrezas prácticas: Utilizamos herramientas como Class Room, que nos permitió, además, de los encuentros presenciales, sistematizar nuestras reuniones y avances, construir de manera colectiva nuestras reflexiones, con las cuales se aportó a fortalecer en nuestros estudiantes competencias del trabajo en equipo y colaborativo, juicio analítico y crítico, toma de decisiones, sentido ético, destrezas prácticas para

\footnotetext{
${ }^{6}$ Adelantar una propuesta de Litigio de Alto Impacto, implica contar con recursos económicos necesarios, los cuales se han buscado a partir de la inscripción de proyectos de investigación.

${ }^{7}$ Inició paro estudiantil desde el mes de octubre de 2015, lo que frena las actividades programadas.
} 
buscar, generar, sistematizar y analizar la información, además del despliegue de creatividad para asumir las tareas y retos propuestos.

Desde el eje trato comunitario: Se avanzó en el análisis previo de los prejuicios y romanticismos con los que nos enfrentamos a algunas personas o grupos de personas, haciendo ejercicio de verbalizarlos previamente y luego confrontarlos con el trabajo de campo. Esto nos permitió comprender la pluralidad de saberes que nos enriquecen y que a veces son despreciados por la academia, logrando comprender el significado de la fidelidad, la privacidad y la confidencialidad como parte del respeto a las personas a su autonomía y su libertad. Es decir, que evidenciamos como se conjuga la racionalidad, las emociones, las pasiones y los sentimientos (Camps, 2011)

Desde el eje Investigativo: Avanzamos en las búsquedas individuales y colectivas de los estudiantes, entendimos que el conocimiento no está dado, no es estático, es necesario construirlo, desde y para el otro, entender que, si se desean soluciones acordes al contexto y autosostenibles. Avanzando hacia "la educación liberadora, problematizadora, ya no puede ser el acto de depositar, de narrar, de transferir o de trasmitir "conocimientos" y valores a los educandos, meros pacientes, como lo hace la educación "bancaria", sino ser un acto cognoscente." (Freire, 1970, p. 58)

Desde el Componente Jurídico: El enfoque del derecho cambia y se dimensiona, es social y cultural, requiere una lectura interdisciplinaria para entender la realidad y que permita darle solución a los problemas que son objeto de estudio, pero comprendiendo que el Derecho, muchas veces no soluciona realidades complejas y que necesariamente se requieren de otras disciplinas, otros cursos de acción y el reconocimiento de otros saberes y lugares de conocimiento.

Desde el componente Político: Asumir temáticas que tienen que ver con las perspectivas de género y la interculturalidad, se convierte en una apuesta política, de un lado porque son temáticas que no se abordan de manera cotidiana en nuestras aulas y de otro, como lo señala Viaña (2008) "Es muy importante discutir con estas corrientes, aunque nos hablen de "inclusión" y de "igualdad", porque están vaciadas en el mismo horizonte del amoldamiento de todos al conjunto de las lógicas liberales y mercantiles, aunque de una forma más "amistosa", que es todavía más nociva por la ilusión de "aliado" y "amigo" que crea." (p. 13), esto nos exige estar atentos para que expresiones como perspectiva de género, intercultural, diálogo de saberes, respeto cultural, no se conviertan en simples muletillas de moda, que engalanan nuestros escritos, pero que 
carecen de una comprensión dado su carácter polisémico y la posibilidad de concretarlas en nuestras prácticas académicas.

Desde el componente de la Comunicación: Se requieren estrategias que incidan e informen a la opinión pública del trabajo realizado, como un compromiso ético de socialización del conocimiento y de transparencia de nuestro trabajo. "Los hombres no se hacen en el silencio, sino en la palabra, en el trabajo, en la acción, en la reflexión" (Freire, 1970, p. 69)

En este sentido, nuestros estudiantes diseñaron y grabaron dos (2) programas de radio, para ser emitidos en la Emisora de la Universidad de Antioquia y en la Emisora del Resguardo: Protección Jurídica a la Propiedad Intelectual Indígena, donde concluyen que es necesario que sean los pueblos desde su posibilidad de autodeterminación, quienes avancen en la definición de esta protección, ya que entra en tensión lo individual con lo colectivo, las lógicas de mercado, con las lógicas de los pueblos originarios y Maquilas, una opción de trabajo digno, en el cual proponen la necesidad de organización comunitaria, en pro de utilizar la normatividad existente, para autogestionar y defender los intereses colectivos de la comunidad, en busca de ingresos y trabajo en condiciones dignas, según lo propuesto por la Organización Internacional del Trabajo.

Desde el eje profesional: Se aportó a la formación integral de nuestros estudiantes, a la sensibilización sobre las diferentes formas de ejercer como abogados(as), la importancia de la integridad como parte fundamental de la estructuración profesional y habilidades para enfrentar los dilemas éticos y los juicios sobre la responsabilidad de la mitigación de los riesgos y la acción sin daño.

Desde el eje de transformación comunitaria: Primero es necesario transformarnos, ocupar nuevamente nuestro lugar de sujetos, entender que hay otras formas de enseñar el derecho, otras formas de ejercer el derecho, otras realidades, otros conocimientos válidos, la meta es lograr "litigio estratégico de alto impacto", pero a este sólo se llega después de un camino recorrido en el que la palabra litigio cambia de significado, va más allá de las estructuras jurídicas, y por tanto, admite formación jurídico-política de las comunidades, movilización social, solidaridad, asociación, empoderamiento, diálogo y soluciones que no necesariamente pasan por el derecho. 
Exige confianza y creatividad, como motores de la transformación: creer que es posible y hacerlo de manera diferente, para avanzar en transformaciones hacia sociedades justas, políticas, éticas y de reconocimiento a las culturas, que permitan una educación emancipatoria, que rompa con su función de dominación y alienación, que evidencien las tensiones, seguimos abordando nuevas teorías con viejos moldes, es decir, con estructuras culturales, llenas de eufemismos y sofismas. "Las personas producidas en esta "cultura" difícilmente podrán siquiera plantearse el "diálogo", el "respeto" y la "convivencia"” (Viaña, 2008, p. 36). Por lo tanto, cuando como profesores, logramos entender la situación de los estudiantes y la nuestra, aceptamos que el "otro", el estudiante, es un interlocutor válido, podemos avanzar en un compromiso mutuo con la solución de los problemas y la transformación de las comunidades que se intervienen, y de los sujetos que participamos a lo largo de este recorrido, de este tejer experiencias.

\section{Referencias bibliográficas}

Bárcena, F. y Mélich, J-C (2000): La educación como acontecimiento ético. Natalidad, narración y hospitalidad. España: Paidós

Blázquez Martín, D. (2015): Clínicas de derechos humanos. Causas y ventajas del éxito de un modelo de educación jurídica del siglo XXI. En: Revista Educación y Derecho. No. 11. Recuperado de: http://revistes.ub.edu/index.php/RED/article/view/11999/14776

Camps, V. (2011): El Gobierno de las Emociones. España: Herder.

CITI Buenas Prácticas Clínicas, (Curso Virtual-Lección 20) University of Miami-CITI Program. Septiembre de 2012.

CITI Aspectos Básicos en Protección de Sujetos Humanos, (Curso Virtual) University of Miami-CITI Program. Septiembre de 2012.

CITI Biomedical Research, (Curso Virtual) University of Miami-CITI Program. Septiembre de 2012.

Colombia, Congreso de la República (2010): Ley 1374 de 2010 Por medio de la cual se crea el Consejo Nacional de Bioética y se dictan otras disposiciones. Recuperado de: http://www.secretariasenado.gov.co/senado/basedoc/ley_1374_2010.html

Colciencias (2017): Política de Ética, Bioética e Integridad Científica. Recuperado de: https://minciencias.gov.co/sites/default/files/upload/noticias/politica-etica.pdf 
Colciencias (2018): Resolución 314 del 2018 por la cual el Departamento Administrativo de Ciencia, Tecnología e Innovación Colciencias, adopta la Política de Ética de la Investigación, Bioética e Integridad Científica, Recuperado de: https://minciencias.gov.co/normatividad/resolucion-0314-2018

Comisión Nacional para la Protección de los Sujetos Humanos de Investigación Biomédica y del Comportamiento. (1979): Informe Belmont: Principios éticos y orientaciones para la protección de sujetos humanos en la experimentación. Recuperado de: http://www.bioeticayderecho.ub.edu/archivos/norm/InformeBelmont.pdf

Confederación Suiza (sf): Enfoque de Acción sin Daño. Recuperado de: https://www.eda.admin.ch/countries/colombia/es/home/internationale-

zusammenarbeit/projekte/proyectos-cosude/nothilfe/accion_sin_dano.html

Correa, L. y Fergusson, A. M. (Comp.). (2008): La Enseñanza Clínica del Derecho. Medellín: Fundación Universitaria Luis Amigó.

Cortina, A. (2016): ¿Para qué sirve realmente la ética? España: Editorial Paidós.

Courtis, C. (2007): Educación Clínica como práctica transformadora. En: M. Villarreal y C. Courtis (Coord), Enseñanza Clínica del Derecho. Una alternativa a los métodos tradicionales de formación de abogados. pp. 9-24, México: Sans Serif Editores.

De Sousa Santos, B. (2014): Más allá del pensamiento abismal: de las líneas globales a una ecología de saberes. En B. De Sousa Santos y M. P. Meneses (Eds.) Epistemologías del Sur (perspectivas) (pp. 21 - 66). España: Ediciones Akal.

Denzin, N. y Lincoln, Y. (2012): Controversias paradigmáticas, contradicciones y confluencias emergentes. En N. Denzin, y Y. Lincoln (Coord.), El campo de la investigación Cualitativa. Manual de investigación cualitativa Vol. I (pp. 43 - 102). Barcelona: Gedisa.

Feito, L. (2007): Vulnerabilidad. Anales del Sistema Sanitario de Navarra, 30 (Supl. 3), 07-22. Recuperado de: http://scielo.isciii.es/scielo.php?script=sci_arttext\&pid=S1137$66272007000600002 \& \operatorname{lng}=$ es\&tlng=es.

Freire, P (1970): Pedagogía del Oprimido (3era. Ed. 2010) Recuperado de: http://www.ensayistas.org/critica/liberacion/varios/freire.pdf 
Gallego Marín, J. A. (2012): Cuadernos de metodología de la investigación para la escuela de derecho: Líneas básicas para direccionar el qué hacer de la investigación. Pereira: Universidad Libre de Pereira.

Giraldo Ángel, J. (2012): Metodología y Técnica de la Investigación Jurídica. Ibagué: Universidad de Ibagué.

Giraldo Ángel, J. (2012): Metodología y Técnica de la Investigación Sociojurídica. Ibagué: Universidad de Ibagué.

Rodríguez, G. (2007): Prólogo. En: M. Villarreal y C. Courtis (Coord), Enseñanza Clínica del Derecho. Una alternativa a los métodos tradicionales de formación de abogados. pp. 5-7, México: Sans Serif Editores.

Smith, L. T. (2012): Caminando sobre terreno resbaladizo. La investigación de los pueblos nativos en la era de la incertidumbre. En: Denzin y Lincon (coord.) Manual de Investigación Cualitativa. Vol 1. España: Gedisa

UNESCO (2005): Declaración Universal sobre Bioética y Derechos Humanos. Recuperado de: http://portal.unesco.org/es/ev.phpURL_ID=31058\&URL_DO=DO_TOPIC\&URL_SECTION=201.html

Universidad de Antioquia. (2014): Resolución Rectoral 40455 Por la cual se crea el Comité Central de Ética en Investigación de la Universidad de Antioquia -CCEI. Recuperado de: www.udea.edu.co.

Universidad de Antioquia (2015): Manual de Funcionamiento de los Comités de Ética en Investigación de la Universidad de Antioquia. Recuperado de: www.udea.edu.co

Universidad de Antioquia (2018): Comité para el Desarrollo de la Investigación CODI. Recuperado de: www.udea.edu.co

Viaña, J. (2008): Reconceptualizando la Interculturalidad. En: J. Viaña y L. Tapia y C. Walsh. Construyendo Interculturalidad Crítica. Lima, Perú: III-CAB. P 9-61.

Witker, J. (2008): Hacia Una Investigación Jurídica Integrativa. En Boletín Mexicano de Derecho Comparado, No. 122, pp. 943-964. 